\title{
Style Versus Substance: Multiple Roles of Language Power in Persuasion
}

\author{
JOHN R. SPARKS \\ University of Dayton
}

\author{
Charles S. Areni \\ University of Sydney
}

\begin{abstract}
This research explores how message style influences persuasion in conjunction with message substance. Using the elaboration likelihood model, the study operationalizes message style as language power and message substance as argument quality, then considers the multiple roles language power can assume in persuasion. The authors investigate whether language power acts as a (a) central argument, (b) peripheral cue, (c) biasing influence on assessment of arguments, or (d) distraction that inhibits argument processing. Additionally, they manipulate exposure time to examine how processing ability influences which persuasive roles language power assumes. The authors find empirical support for the multiple-roles perspective and conclude that the role of message style depends partially on the ability to process message details.
\end{abstract}

Is it what you say or how you say it? Asserting that a speaker's persuasive ability arises both from message substance and presentation style would be axiomatic at best. However, in applications ranging from politics to law, from religion to marketing, the basic question of how style versus substance affects persuasion encompasses many complex and interesting issues. For example, in some contexts, style may actually become substance, rendering the effects of the two largely indistinguishable. In some contexts, style may be used to infer source characteristics, which in turn affect perceptions of message substance. In other contexts, style may inhibit or enhance the accurate processing of message substance, while in still other contexts, style may provide a convenient means of simply surmising message substance, even with little actual knowledge of it. Collectively, these possibilities suggest that, depending on context, speaking style may assume multiple roles in persuasion. The purpose of the present research is to investigate multiple persuasive effects of speaking style under conditions of low versus high ability to process message content.

Drawing from the communications literature, we examine speaking style in terms of language power, which is a construct that associates the presence of certain speech markers with the perceived social power of speakers. By studying courtroom transcripts, O'Barr (1982) noted differences in the speech patterns of individuals participating in legal proceedings, and suggested that these differences were associated with the social status of participants. Based on these observations, O'Barr classified these speech markers into powerless 
and powerful language. Typical, powerless language contains the frequent use of nonverbal hesitations (e.g., "uhhh," "errr"), verbal hesitations (e.g., "you know," "I mean"), deictic phrases (e.g., "that one there"), tag questions (e.g., "That's how it happened, isn't it?"), hedges ("I guess," "kind of"), and intensifiers (e.g., "He really did," "It was very, very dark."). In contrast, speakers using powerful language exhibited an absence of these markers. Subsequent language-power research has identified tag questions, hedges, verbal hesitations, and nonverbal hesitations as the strongest indicators of powerless language (Bradac \& Mulac, 1984a, 1984b). Since that time, research has tended to focus on these markers as exemplifying powerless language (Gibbons, Busch, \& Bradac, 1991; Holtgraves \& Lasky, 1999; Hosman \& Wright, 1987).

Research on language power and source perceptions has clearly established that speakers using powerful language are rated more positively in terms of intelligence, attractiveness, competence, credibility, sociability, and social power than are speakers using powerless language (Bradac \& Mulac, 1984a, 1984b; Erickson, Lind, Johnson, \& O'Barr, 1978; Hosman \& Wright, 1987; O’Barr, 1982). Surprisingly, early research did not establish an effect for language power on persuasion (Gibbons et al., 1991). Sparks, Areni, and Cox (1998) attributed this result to the common use of written transcripts as stimuli in language-power research. They found that language power affected persuasion for participants exposed to an audiotaped or videotaped presentation, but not for participants who read written transcripts of the same presentation. However, Sparks and Areni (2002) and Areni and Sparks (2005) later found that language power influenced persuasion when written transcripts were used as experimental stimuli.

These somewhat inconsistent findings suggest one or both of two possibilities. First, powerless speech markers presented in taped stimuli may be more salient than those presented in transcribed stimuli, making them more influential (Chaiken \& Eagly, 1976, 1983). Second, the ability to process message details may be greater with transcribed stimuli than with taped stimuli because message recipients can control their reading speed and can reexamine various sections of the message, as needed. This would allow message recipients to focus more on the substance of the arguments than on speech markers, hence making language power less influential on persuasion (Areni \& Sparks, 2005; Sparks \& Areni, 2002; Sparks et al., 1998). In the present research, we test the latter of these two explanations by using an alternative manipulation of ability to process: time of exposure to printed transcripts.

In general, we predict that the effect of language power on persuasion will be more pronounced when individuals have shorter, as opposed to longer exposure to transcripts of persuasive communications. However, we specify 
alternative persuasive roles of language power when ability is relatively high. Drawing on the elaboration likelihood model (ELM) of persuasion, we propose that language power can affect persuasion in several different ways, depending on the strength of the arguments contained in the persuasive communication and the ability of message recipients to consider the arguments when forming their opinions.

\section{Elaboration Likelihood Model of Persuasion}

Briefly, the ELM theorizes that persuasion occurs along a continuum, anchored at one end by peripheral processing and at the other end by central processing (Petty \& Cacioppo, 1986). When motivation and ability to process information are both high, message recipients elaborate on message content. Persuasion occurs through central processing when message recipients carefully consider the arguments and evidence contained in the message and form opinions based on that content. In contrast, if either motivation or ability is inhibited, persuasion occurs through peripheral processing. Under these circumstances, elaboration on message arguments is limited, and persuasion occurs through simple cues, such as the persuasion context (e.g., mood at the time of message exposure, pleasant surroundings), features of the communication that are not relevant to message arguments (e.g., an unattractive speaker), or the use of simple decision heuristics (e.g., "Experts are always right," "You get what you pay for").

An important difference between language power and many variables studied as peripheral cues in tests of the ELM is the integration of language markers into the actual text of presented arguments. Many variables studied as peripheral cues in ELM research are spatially or temporally separated from the central arguments in the communication, which may oversimplify the effects of these variables in persuasion. On the other hand, being enmeshed within the actual arguments in a persuasive communication increases the likelihood that language power may simultaneously act in multiple roles, which suggests greater complexity in distinguishing between the effects of style and substance (Areni \& Sparks, 2005; Sparks \& Areni, 2002). In the following reported research, we consider the effects of style and substance in the context of the ELM by manipulating the quality of the arguments presented in a persuasive communication and by manipulating the language power used to present the arguments.

As noted previously, pre-exposure levels of elaboration - and, hence, the likelihood of central versus peripheral processing - are determined by two classes of variables: those affecting motivation, and those influencing ability. Variables in each class can be further described as being either individual or 
situational sources of influence. Individual influences on motivation to process include, for example, need for cognition and open- versus closedmindedness, whereas the personal relevance of the topic and a forewarning of the intention to persuade represent situational influences on motivation to process. Variables influencing the ability to process can also be either situational (e.g., a distracting stimulus) or individual (e.g., general intelligence; Petty, Priester, \& Wegener, 1994; Petty, Unnava, \& Strathman, 1991).

Situational influences on ability to process have been manipulated by altering the communication medium or modality (Chaiken \& Eagly, 1976, 1983; Sparks et al., 1998), the tape speed of audiotaped messages (Moore, Hausknecht, \& Thamodaran, 1986), or by introducing a distraction task during exposure (Petty, Wells, \& Brock, 1976). However, these approaches are limited because they confound ability with qualitative processing differences in each condition. For example, as noted previously, communication modality manipulations may confound ability with salience of the speaker, drawing attention to the speaker and away from the message itself (Chaiken \& Eagly, 1983). Likewise, the specific nature of a distraction task may prime concepts or stimuli related to the message content (Heil \& Rolke, 2004). Finally, tape-speed manipulations alter voice pitch and syllable timing, variables known to influence reactions to spoken communications (Bradac \& Street, 1990). In the present research, we avoid these potential confounds by manipulating ability through the time given to process printed transcripts.

The ELM's basic framework specifies five ways that communication variables such as language power can influence persuasion, which depends on the amount of elaboration during exposure to the communication. When elaboration likelihood is relatively low, communication variables operate as peripheral cues, influencing persuasion in the absence of detailed, topicrelevant thinking. When elaboration likelihood is relatively high, communication variables operate either as a biasing influence on message processing or as a strong or weak argument for accepting or rejecting an advocated position. Finally, when elaboration likelihood is in the moderate range of the continuum, communication variables operate either as elaboration enhancers that provoke topic-relevant thinking or as elaboration inhibitors that prevent audiences from focusing on message content.

However, actual empirical research has suggested that variables that enhance versus inhibit elaboration are constrained by ceiling versus floor effects, respectively. That is, message variables enhance elaboration mainly when elaboration likelihood is otherwise low (Maheswaran \& Chaiken, 1991; Thompson, Chaiken, \& Hazlewood, 1987), whereas variables inhibit elaboration mainly when elaboration is otherwise high (Munch \& Swasy, 1988; Petty et al., 1976). Given the ambiguous status of effects in the moderate range of the elaboration continuum, we focus on possible roles for language 
power under low and high levels of elaboration likelihood: as a peripheral cue, a biasing influence, and as an inhibiting influence.

\section{Multiple Roles of Language Power}

\section{Language Power as a Peripheral Cue}

Previous research on language power has suggested that it acts as a peripheral cue under conditions that limit the ability to process message details. When ability is limited, message recipients may not be able to scrutinize relevant arguments and evidence to arrive at carefully reasoned opinions. Instead, language power may serve as an easily processed cue, suggesting a confident and trustworthy speaker versus an uncertain and unreliable speaker. The cue, in turn, provides the basis for forming an attitude about the message itself.

Sparks et al. (1998), for example, found that language power exerted a stronger effect on persuasion when the communication was presented in a taped, rather than printed format. Argument quality played the dominant role in influencing attitudes in the latter case (see also Sparks \& Areni, 2002). This difference can be accounted for by the limited situational ability afforded by taped stimuli relative to printed stimuli, which can be read carefully and reread if necessary (MacInnis, Moorman, \& Jaworski, 1991). To the extent that limited ability leads message recipients to base attitudes more on the speech markers associated with powerless language and less on argument quality, then language power may act as a peripheral cue. This suggests the following hypotheses:

Hypothesis $1 a$. When the ability to process message details is relatively low, message recipients will form less favorable attitudes toward the speaker's advocated position when the speaker uses powerless language than when the speaker uses powerful language.

Hypothesis $1 b$. When the ability to process message details is relatively low, speaker-related thoughts generated by message recipients will be more negative when the speaker uses powerless language than when the speaker uses powerful language.

Hypothesis 1c. When the ability to process message details is relatively low, language power will have little or no effect on the valence of argument-related thoughts generated by message recipients. 
Hypothesis $1 d$. When the ability to process message details is relatively low, the effects of language power on attitudes toward the speaker's advocated position will be mediated by the valence of speaker-related thoughts and not by argument-related thoughts.

\section{Language Power as a Biasing Influence}

Language power may also influence persuasion when ability is relatively high, not as a relevant argument, but by biasing an audience's evaluation of relevant arguments. This is because language power is enmeshed in the actual message content. There are few obvious perceptual differences between the style and the substance of the message. This makes it more difficult for message recipients to separate the effects of the former from the latter, resulting in a biasing influence.

Consistent with this reasoning, several studies of language power have concluded that powerless language creates the impression that a speaker lacks confidence in his or her own arguments (Bradac \& Mulac, 1984a, 1984b), which leads message recipients to counterargue the speaker's position (Sparks \& Areni, 2002). However, unlike acting as a peripheral cue, the biasing influence occurs because message recipients elaborate on relevant arguments sufficiently to engage in some degree of counterargumentation. Hence, we expect that attitudes will be less favorable when a speaker uses powerless language than when a speaker uses powerful language, even when message recipients are relatively high in ability to process message content.

Moreover, to the degree that message recipients counterargue the speaker's advocated position, argument-related thoughts should be more negative when powerless as opposed to powerful speech is used. Powerless language should also produce more negative speaker-related thoughts. However, individuals who exert the cognitive effort to elaborate on the substance of a communication will base their attitudes on argument-related thinking, rather than on thoughts about the speaker's style. That being the case, then, argument-related thoughts and not speaker-related thoughts will mediate the causal sequence leading from language power to attitude. This suggests the following hypotheses:

Hypothesis $2 a$. When the ability to process message details is relatively high, message recipients will form less favorable attitudes when the speaker uses powerless language than when the speaker uses powerful language.

Hypothesis $2 b$. When the ability to process message details is relatively high, argument-related thoughts generated by 
message recipients will be more negative when the speaker uses powerless language than when the speaker uses powerful language.

Hypothesis 2c. When the ability to process message details is relatively high, speaker-related thoughts generated by message recipients will be more negative when the speaker uses powerless language than when the speaker uses powerful language.

Hypothesis $2 d$. When the ability to process message details is relatively high, the effects of language power on attitudes toward the speaker's advocated position will be mediated by the valence of argument-related thoughts and will not be mediated by the valence of speaker-related thoughts.

\section{Language Power as a Distracting Influence}

Powerless language may also distract audiences from processing message content when the ability to process is otherwise unlimited. According to Bradac and Street (1990) and Sparks et al. (1998), powerless speech markers may actually interrupt the flow of discourse in a communication setting, which may distract message recipients from processing the arguments contained in the message. Areni and Sparks (2005) argued that powerless language redirects message recipients' thoughts toward the speaker, perhaps in order to explain the speaker's apparent lack of confidence or certainty. Along these lines, Gibbons et al. (1991) and Sparks and Areni (2002) reported that message recipients generated fewer argument-related thoughts and more speaker-related thoughts when speakers used powerless language, rather than powerful language. Moreover, Sparks and Areni found that the effect of argument quality on persuasion was reduced when the speaker used powerless language, as opposed to powerful language, again suggesting that powerless language markers interrupted argument-related processing. This suggests the following hypotheses:

Hypothesis $3 a$. When the ability to process message details is relatively high, message recipients will generate more speakerrelated thoughts when the speaker uses powerless language than when the speaker uses powerful language.

Hypothesis $3 b$. When the ability to process message details is relatively high, message recipients will generate fewer argument-related thoughts when the speaker uses powerless language than when the speaker uses powerful language. 
Hypothesis $3 c$. When the ability to process message details is relatively high, the effect of argument quality on the valence of argument-related thoughts will be less pronounced when the speaker uses powerless language, as opposed to powerful language.

Hypothesis $3 d$. When the ability to process message details is relatively high, the effect of argument quality on attitudes toward the advocated position will be less pronounced when the speaker uses powerless language, as opposed to powerful language.

\section{Method}

The hypotheses were tested using a 2 (Ability: low vs. high) $\times 2$ (Language: powerful vs. powerless) $\times 2$ (Argument: strong vs. weak) full-factorial experimental design. Participants were 118 undergraduate business students from a Midwestern university, who participated in the research in exchange for extra course credit. The participants were randomly assigned to treatment groups.

\section{Procedure}

Participants were told that they would review a verbatim transcript from a commercial testimonial for the Scann-X computer scanner, the hypothetical focal product. Early language-power research established the norm of using transcribed stimuli in an effort to protect internal experimental validity. As noted previously, the language-power variable is defined as the presence or absence of powerless speech markers and, therefore, pertains exclusively to the actual words and utterances of the speaker, rather than on his or her vocal characteristics (e.g., pitch, volume, accent).

Using this view to support the use of written transcripts instead of audio or video recordings, Bradac and Mulac (1984b) argued that recordings contain noise, which "is produced by idiosyncratic aspects of voice quality, intonation, etc., and by variations in vocal presentation from one message version to another" (p. 310). Such variation confounds researchers' ability to attribute effects to language power and not the idiosyncratic vocal characteristics present on recordings. In addition, taped stimuli may enhance the salience of powerless speech, potentially confounding the effects of processing ability. Hence, while taped stimuli have been used successfully in past 
research, our focus on manipulating a situational influence on the ability to process led us to rely on transcribed stimuli in the present experiment.

In all conditions, the experimenter entered the room and distributed packets that contained the stimuli, instructions, and questionnaires. Research participants were asked to keep the packets face down until they were instructed otherwise. When all participants received their packets, they were told to turn to the first page and read the instructions while the experimenter read them aloud. Participants were told they would read a "verbatim transcript of a person giving a testimonial about a computer product." They were to evaluate the testimonial on a number of different dimensions, including its suitability for use in an actual advertisement. After all of the participants finished reading the transcripts, they were instructed to complete the questionnaires.

The transcripts themselves were formatted to appear similar to those used in video production, with each page divided into two columns. The righthand column contained the actual transcribed testimonial, while the lefthand column contained four very brief descriptions of what would have been on the screen had the participants been actually watching the testimonial. For example, the video description opened with "man seated at desk with scanner" and concluded with "camera zooms to scanner on desk, shows logo."

\section{Independent Variables}

Ability to process. Participants in the high-ability condition were given $5 \mathrm{~min}$ to read the transcript, which was approximately 600 words in length, including the video descriptions and exclusive of any powerless speech markers. The weak-argument version totaled 591 words, while the strongargument version contained 580 words. The 5 -min time period was designed to permit ample time to read carefully, process message content, and scrutinize relevant arguments. Participants in the low-ability conditions were given $20 \mathrm{~s}$ to read the transcript. Prior to exposure, they were told that they would receive very little time to read the transcript, so they should read it quickly. We informed participants of the time limitation in advance in order to encourage them to go over the entire transcript quickly, rather than start slowly and be forced to stop after only a few sentences had been read.

Language power. As with previous research, we manipulated language power through the presence or absence of powerless speech markers. The language in the powerless condition contained speech markers commonly associated with powerless language, including 6 tag questions (e.g., ". . . isn't it?”, “. . . right?”), 12 hedges (e.g., "I guess," "I think”), 18 verbal hesitations 
(e.g., "you know," "Let's see now"), and 12 nonverbal hesitations (e.g., "um," "ah"). These markers were omitted from the powerful version of the testimonial (O’Barr, 1982).

Argument quality. Previous manipulations of argument quality have relied heavily on pretest results to establish strong versus weak arguments, leaving underlying conceptual issues largely unexplored (Areni, 2002, 2003). However, a closer examination of the resulting manipulations suggests that most have focused on altering (a) the attributes or benefits associated with a product (Petty, Cacioppo, \& Goldman, 1981; Petty, Cacioppo, \& Schumann, 1983); (b) performance levels of a product on a given set of attributes (Areni \& Sparks 2005; Wheeler, Petty, \& Bizer, 2005); or (c) structural aspects of the argument linking one statement to another (Munch, Boller, \& Swasy, 1993; Heller \& Areni, 2004).

A review of numerous magazine ads for scanners and other computer products eliminated the structural approach on the basis of face validity: Actual ads do not present extended arguments with several interconnected statements. Rather than manipulating the attributes used to describe the product, and perhaps creating qualitative differences in the perception of the product, we manipulated performance levels on a given set of attributes. Hence, the strong arguments stated that the Scann-X, (a) scans color documents in 5 to 10 seconds; (b) perfectly captures colors and details; (c) comes with five photo-editing software packages; (d) automatically adjusts for transparent media; (e) is easily installed in minutes; (f) has 24-hour, 7-day toll-free technical help by phone; (g) costs less than \$200; and (h) comes with a 5-year warranty. By contrast, the weak arguments stated that the scanner, (a) scans in 5 to 10 minutes; (b) clearly captures black-and-white graphics; (c) offers a photo-editing software package for $\$ 150$ extra; (d) requires an additional cover for transparent media; (e) can be installed in about an hour; (f) has a "900" technical-assistance phone number available from 9 a.m. to 5 p.m. weekdays; (g) costs $\$ 300$; and (h) comes with a 30-day warranty.

\section{Dependent Variables}

Attitude toward the product. We measured product attitude using the question "If you were in the market for a new scanner, how would you rate the purchase of the Scann-X?" Participants responded on six 7-point scales ranging from 1 to 7 with the following anchors: undesirablel desirable, harmfullbeneficial, badlgood, foolish/wise, unfavorablelfavorable, and negativelpositive. To assess the scale's unidimensionality, we factoranalyzed the six items using the principal factor method of estimation. The analysis returned a single factor, with all loadings exceeding 0.80 . We assessed 
scale internal consistency with Cronbach's alpha, which equaled 0.96. Based on these results, we concluded that the attitude measure performed satisfactorily. Participant attitude was calculated as the average of the six items.

Valenced thought indexes. Data collection included a thought-listing exercise in which participants were asked to "list any and all thoughts you had while reading the endorser's testimonial. These can include things related to the speaker, the actual product, or anything else you might have thought about-including things completely unrelated to the testimonial." To encourage distinct cognitive responses, participants were asked to list each thought on a separate line.

Two coders who were blind to the research hypotheses - one a professional market researcher, and the other a postgraduate research assistantassigned cognitive responses according to six categories: (a) positive messagerelated thoughts; (b) negative message-related thoughts; (c) positive thoughts about the speaker; (d) negative thoughts about the speaker; (e) other positive thoughts; and (f) other negative thoughts. Interrater agreement was $81 \%$ across all categories. Discrepancies were resolved via discussion between the coders.

Following Greenwald (1968), we calculated the valenced index for message-related cognitive responses as the difference between the number of positive argument-related thoughts and the number of negative argumentrelated thoughts. Similarly, the valenced index for speaker-related cognitive responses was calculated as the difference between positive and negative speaker-related thoughts.

Extent of message-related and speaker-related thinking. We operationalized the extent of participants' message-related thinking as the total number of message-related thoughts. Likewise, the extent of speaker-related thinking was operationalized as the total number of speaker-related thoughts. The means, standard deviations, and cell sizes for all dependent variables in all experimental conditions are presented in Table 1 .

\section{Results}

\section{Manipulation Checks}

Argument quality. To assess the success of the argument quality manipulation, we asked participants to "rate the content of the endorsement" on a three-item 7-point scale ranging from 1 to 7 with the following anchors: invalid arguments/valid arguments, unpersuasive/persuasive, and unconvincing/ convincing. The scale exhibited adequate internal consistency $(\alpha=.94)$, so items were averaged to produce a composite evaluation of argument quality. 
Table 1

Cell Sizes and Means of Study Variables

\begin{tabular}{|c|c|c|c|c|c|c|}
\hline \multirow[b]{2}{*}{ Variable } & \multicolumn{3}{|c|}{$\begin{array}{l}\text { High processing } \\
\text { ability }\end{array}$} & \multicolumn{3}{|c|}{$\begin{array}{c}\text { Low processing } \\
\text { ability }\end{array}$} \\
\hline & $N$ & $M$ & $S D$ & $N$ & $M$ & $S D$ \\
\hline \multicolumn{7}{|l|}{ Attitude toward the proposal ${ }^{\mathrm{a}}$} \\
\hline Strong argument/Powerful language & 15 & 5.68 & 0.39 & 15 & 5.13 & 0.80 \\
\hline Strong argument/Powerless language & 15 & 4.42 & 1.44 & 13 & 4.05 & 1.26 \\
\hline Weak argument/Powerful language & 15 & 3.70 & 1.18 & 15 & 3.63 & 0.95 \\
\hline Weak argument/Powerless language & 14 & 3.04 & 1.28 & 14 & 3.44 & 1.05 \\
\hline \multicolumn{7}{|l|}{ Valenced argument-related index ${ }^{\mathrm{b}}$} \\
\hline Strong argument/Powerful language & 15 & 1.20 & 1.32 & 15 & 0.73 & 1.39 \\
\hline Strong argument/Powerless language & 15 & 0.13 & 1.19 & 13 & -0.15 & 0.89 \\
\hline Weak argument/Powerful language & 15 & -3.07 & 2.12 & 15 & -0.67 & 1.18 \\
\hline Weak argument/Powerless language & 14 & -1.21 & 1.93 & 14 & -0.71 & 1.20 \\
\hline \multicolumn{7}{|l|}{ Valenced speaker-related index ${ }^{\mathrm{c}}$} \\
\hline Strong argument/Powerful language & 15 & 0.13 & 0.74 & 15 & 0.07 & 0.46 \\
\hline Strong argument/Powerless language & 15 & -1.53 & 1.25 & 13 & -0.62 & 1.04 \\
\hline Weak argument/Powerful language & 15 & -0.40 & 1.12 & 15 & -0.07 & 0.80 \\
\hline Weak argument/Powerless language & 14 & -2.57 & 1.95 & 14 & -1.29 & 1.33 \\
\hline \multicolumn{7}{|l|}{ Extent of argument-related thinking ${ }^{\mathrm{d}}$} \\
\hline Strong argument/Powerful language & 15 & 3.07 & 2.02 & 15 & 1.73 & 1.28 \\
\hline Strong argument/Powerless language & 15 & 1.13 & 0.92 & 15 & 1.15 & 1.34 \\
\hline Weak argument/Powerful language & 15 & 4.00 & 2.04 & 15 & 0.73 & 1.16 \\
\hline Weak argument/Powerless language & 14 & 1.71 & 1.64 & 14 & 0.79 & 1.19 \\
\hline \multicolumn{7}{|l|}{ Extent of speaker-related thinking ${ }^{\mathrm{e}}$} \\
\hline Strong argument/Powerful language & 15 & 0.53 & 0.74 & 15 & 0.40 & 0.63 \\
\hline Strong argument/Powerless language & 15 & 2.53 & 1.51 & 15 & 0.92 & 1.50 \\
\hline Weak argument/Powerful language & 15 & 0.87 & 1.19 & 15 & 0.40 & 0.82 \\
\hline Weak argument/Powerless language & 14 & 3.21 & 1.97 & 14 & 1.92 & 1.27 \\
\hline
\end{tabular}

${ }^{a}$ Mean of undesirableldesirable, harmfullbeneficial, bad/good, foolish/wise, unfavorablel favorable, and negative/positive. ${ }^{\mathrm{b}}$ Difference between positive and negative argumentrelated thoughts. ${ }^{\mathrm{C}}$ Difference between positive and negative speaker-related thoughts. ${ }^{\mathrm{d}}$ Total number of argument-related thoughts. ${ }^{\mathrm{e}}$ Total number of speaker-related thoughts. 
A three-way ANOVA with the manipulation-check measure as dependent variable and argument quality, language power, and ability to process as independent variables produced a significant argument quality main effect, supporting a successful manipulation, $F(1,108)=23.54, p<.001, \omega^{2}=.14$. Participants who were exposed to weak arguments rated them lower $(M=$ $3.01)$ than did participants who were exposed to strong arguments $(M=4.06)$.

Language power. To evaluate the language-power manipulation, we relied on the well-established relationship between powerless language and negative audience perceptions of the speaker (Bradac \& Mulac, 1984a, 1984b; Erickson et al., 1978; Gibbons et al., 1991; Hosman \& Wright, 1987; O'Barr, 1982). Participants gave their perceptions of the speaker's language on a three-item 7-point scale ranging with the following anchors: timidl assertive, powerless/powerful, and weak/strong. Cronbach's alpha for these items was .92. The items were averaged for a composite measure of language power.

This manipulation-check measure was used as the dependent variable in a three-way ANOVA with argument quality, language power, and ability to process as independent variables. In support of a successful manipulation, the results show a significant language power main effect, $F(1,108)=39.88$, $p<.001, \omega^{2}=.23$. Participants in the powerless language condition rated the language power lower $(M=3.03)$ than did those in the powerful language condition $(M=4.50)$.

Ability to process. On the premise that greater elaboration produces greater mastery of the arguments contained in a persuasive communication, we tested participant recall with a series of five multiple-choice questions relating to the arguments contained in the weak and strong testimonials. Recall was calculated as the total number of correct responses. This total served as the dependent variable in a three-way ANOVA with argument quality, language power, and ability as independent variables. The significant main effect of ability supported a successful manipulation, $F(1,108)=$ $180.71, p<.001, \omega^{2}=.51$. Participants in the low-ability condition correctly answered an average of 1.1 questions, while those in the high-ability condition correctly answered an average of 3.4 questions.

Our decision to warn the low-response ability participants of the limited time available to read the transcripts was done to minimize any ordering effects that might occur from not completing the entire transcript. Such an effect would favor recall of early arguments. Having taken steps to avoid it, we felt it prudent to check for the effect. Examination of correct responses suggested that our efforts were not entirely successful. Participants in the low-response ability conditions tended to answer correctly questions pertaining to the first two arguments. Therefore, we must acknowledge the possibility that an ordering effect may represent a potential source of bias. 
While a point of some concern, the data offer good reason to believe that, if present, the ordering effect did not bias either the argument quality or language power manipulations. Our check of the argument quality manipulation reported previously did not produce a significant Argument Quality $\times$ Processing Ability interaction, $F(1,108)=2.33, p>.10$, which suggests that participants in the low processing ability did not differ from the high-ability participants in their assessments of the arguments. In other words, respondents in the low processing ability conditions clearly recognized the weak arguments as such. Similarly, a Processing Ability $\times$ Language Power interaction did not emerge in the language power manipulation check reported previously, $F(1,108)=2.02, p>.10$, suggesting that participants in the low processing ability conditions recognized the powerless speech markers in the transcripts they read. On the whole, the data appear to support a successful, unbiased processing ability manipulation.

\section{Hypothesis Tests}

Hypothesis 1a predicted that when ability was low, message recipients would form less favorable attitudes toward the product when the speaker used powerless rather than powerful language. Hypothesis $2 \mathrm{a}$ predicted a similar effect when ability was high. We tested these two hypotheses using a three-way ANOVA, with attitude as the dependent variable and ability, argument quality, and language power as independent variables. As expected, language power exerted a significant main effect on attitude, $F(1$, $108)=15.51, p<.001, \omega^{2}=.08$, with those who were exposed to powerless language expressing more negative attitudes $(M=3.74)$ than those who were exposed to powerful language $(M=4.50)$. The Ability $\times$ Language Power interaction effect did not achieve significance $(F<1)$, indicating that the language-power effect occurred in both the low- and high-ability conditions. Thus, the data support Hypotheses 1a and $2 \mathrm{a}$.

Hypotheses $1 \mathrm{~b}$ and $2 \mathrm{~b}$ predicted that under conditions of low and high ability, respectively, speaker-related thoughts would be more negative when message recipients were exposed to powerless, rather than powerful language. To test these hypotheses, we used a three-way ANOVA with the valenced speaker-related cognitive response index as the dependent variable and ability, argument quality, and language power as independent variables. The results indicate a significant language power main effect on the valenced index, $F(1,108)=44.57, p<.001, \omega^{2}=.25$. However, the Ability $\times$ Language Power interaction also achieved significance, $F(1,108)=5.08, p<.05$, raising the possibility that language power did not significantly affect the valence of speaker-related thoughts in one of the ability conditions. 
Mean contrasts support significant language effects in both ability conditions. However, contrary to expectations, the language power effect was stronger when ability was high: low ability, $F(1,53)=14.23, p<$ $.001, \omega^{2}=.19$; high ability, $F(1,55)=30.93, p<.001, \omega^{2}=.33$. Means in both ability conditions were in the expected direction: low ability, $M_{\text {powerless }}=-0.96, M_{\text {powerful }}=0.00$; high ability, $M_{\text {powerless }}=-0.13, M_{\text {powerful }}=$ -2.03 . Hence, the data support Hypotheses $1 b$ and $2 b$.

Hypothesis 2c predicted that when ability was high, argument-related thoughts would be more negative when a speaker used powerless language than when he or she used powerful language. Hypothesis 1c predicted that when ability was low, this effect would not emerge. These hypotheses were tested using a three-way ANOVA with the valenced message-related index as the dependent variable and ability, argument quality, and language power as independent variables. The results show no significant language power main effect on the valenced message index $(F<1)$, nor was the Ability $\times$ Language Power interaction significant, $F(1,108)=2.51, p>.10$. The data do not support Hypothesis 2c, but they do support Hypothesis 1c, with the qualification that Hypothesis 1c predicted null results.

Hypothesis 1d predicted that language power's effect on attitude would be mediated by the valence of speaker-related thoughts when ability was low, and that its effect on attitude would not be mediated by the valence of message-related thoughts. Following criteria forwarded by Baron and Kenny (1986), a variable mediates the effect of an experimental factor on a dependent variable (a) when the effect of the experimental factor on the mediator is significant; (b) when the mediator correlates with the dependent variable; and (3) when the mediator is introduced to the model as a covariate, the experimental factor's effect on the dependent variable diminishes or disappears.

To test the first criterion of mediation, we used 2 two-way ANOVAs on the low-ability condition with language power and argument quality as independent variables, and attitude and the valenced speaker-related index as dependent variables. With attitude as the dependent variable, we observed a significant language power main effect, $F(1,53)=5.56, p<.05, \omega^{2}=.06$; and likewise with the valenced speaker index as the dependent variable, $F(1$, $53)=14.23, p<.05, \omega^{2}=.19$. Hence, the first criterion for mediation was satisfied. To test the second and third criteria, we used a two-way ANCOVA on the low-ability data with attitude as the dependent variable; argument quality and language power as independent variables; and the valenced speaker index as a covariate. The second criterion requires that attitude and the valenced speaker index correlate significantly. When introduced as a covariate in the model, the valenced speaker index was predictive of attitude, $F(1,52)=4.75, p<.05$, satisfying this criterion. The third criterion-that the 
mediator's presence in the model diminishes or eliminates language power's effect on attitude - was also met. In the presence of the valenced speakerrelated index, language power no longer exerted a significant effect on attitude, $F(1,52)=1.35, p>.20$.

Hypothesis $1 \mathrm{~d}$ also predicted that the valence of argument-related thoughts would not mediate language power's effect on attitude when ability was low. We followed similar procedures as before, only with the valenced message-related index as a covariate. The first criterion described by Baron and Kenny (1986) requires that language power affects the valenced argument-related index. A two-way ANOVA on the low-ability condition with the valenced message-related index as the dependent variable and argument quality and language power as the independent variables showed no significant language power effect on the index, $F(1,53)=2.20, p>.10$. The first mediation criterion was not satisfied. Thus, when ability is low, the valence of speaker-related thoughts (and not argument-related thoughts) mediated language power's effect on attitude. Therefore, the data support Hypothesis 1d.

Hypothesis $2 \mathrm{~d}$ predicted that when ability was high, language power's effect on attitude would be mediated by the valence of argument-related thoughts, and not by speaker-related thoughts. Using the high-ability data, we performed a two-way ANOVA with valenced message index as the dependent variable and argument quality and language power as independent variables. Language power did not significantly affect the valenced-argument index $(F<1)$, failing the first criterion for mediation described previously. Therefore, the data do not support this part of Hypothesis 2d.

Additionally, Hypothesis $2 \mathrm{~d}$ predicted that when ability was high, speaker-related thoughts would not mediate the causal sequence from language power to attitude. Earlier support for Hypotheses $2 \mathrm{a}$ and $2 \mathrm{~b}$ met the first two criteria for mediation (Baron \& Kenny, 1986). Among participants in the high-ability condition, language power exerted significant effects on attitude, $F(1,55)=10.17, p<.01, \omega^{2}=.09$; and on the valenced speakerrelated index, $F(1,55)=30.93, p<.001, \omega^{2}=.33$. The third criterion was tested via a two-way ANCOVA on the high-ability condition with attitude as the dependent variable; language power and argument quality as independent variables; and the valenced speaker-related index as a covariate. With the covariate in the model, language power's effect on attitude only approached statistical significance, $F(1,54)=3.58, p<.10$. Thus, the data support neither prediction in Hypothesis 2d. The effect of language power on attitude was mediated by speaker-related thoughts, regardless of message recipients' ability to process message details.

Hypotheses $3 \mathrm{a}$ and $3 \mathrm{~b}$ predicted that, when ability was high, powerless language would direct thoughts toward the speaker and away from relevant 
arguments. Consequently, message recipients would report fewer messagerelated thoughts (Hypothesis 3a) and more speaker-related thoughts (Hypothesis $3 \mathrm{~b}$ ) when the speaker used powerless language. To test these hypotheses, we conducted 2 three-way ANOVAs with ability, argument quality, and language power as independent variables; and the total number of message-related cognitive responses and speaker-related cognitive responses as dependent variables. With respect to number of speakerrelated cognitive responses, the results show a significant language power main effect, $F(1,108)=46.32, p<001, \omega^{2}=.42 ;$ and a significant Ability $\times$ Language Power interaction, $F(1,108)=5.96, p<.05, \omega^{2}=.05$.

Planned mean contrasts show language power affecting the number of speaker-related cognitive responses in both ability conditions, though the effect was considerably stronger in the high-ability group: high ability, $F(1$, $55)=34.96, p<.001, \omega^{2}=.63$; low ability, $F(1,53)=12.56, p<.001, \omega^{2}=.22$. Message recipients generated more speaker-related thoughts when they were exposed to powerless, rather than powerful language: low ability, $M_{\text {powerless }}=0.96, M_{\text {powerful }}=1.23$; high ability, $M_{\text {powerless }}=1.41, M_{\text {powerful }}=3.53$. Therefore, these results support Hypothesis $3 \mathrm{a}$.

With the number of argument-related cognitive responses as the dependent variable, the results show a significant language power main effect, $F(1$, $108)=18.07, p<.001, \omega^{2}=.16$; and a significant Ability $\times$ Language Power interaction, $F(1,108)=10.93, p<.01, \omega^{2}=.09$. Mean contrasts reveal that the language power effect was significant when ability was high, $F(1$, $55)=22.33, p<.001, \omega^{2}=.39$; but not when it was low $(F<1)$. Among message recipients in the high-ability condition, those who were exposed to powerless language generated fewer message-related cognitive responses $(M=1.41)$ than did those who were exposed to powerful language $(M=3.53)$. Thus, the data support Hypothesis $3 \mathrm{~b}$.

Hypotheses 3c and 3d essentially predicted an Argument Quality $\times$ Language Power $\times$ Ability interaction effect for the valenced message-related cognitive response index and attitude toward the product, respectively. Both predicted interactions should produce an Argument Quality $\times$ Language Power interaction effect in the high-ability condition, such that the effect of argument quality would be smaller in the powerless language condition. With respect to Hypothesis $3 \mathrm{c}$, the results of a three-way ANOVA with ability, argument quality, and language power as independent variables and the valenced message-related cognitive response index as the dependent variable produced a significant three-way Ability $\times$ Argument Quality $\times$ Language Power interaction effect, $F(1,108)<3.67, p<.05$, $\omega^{2}=.01$. Moreover, in the high-ability condition, a two-way ANOVA yielded a significant Argument Quality $\times$ Language Power interaction, $F(1,55)$ $=11.11, \mathrm{p}<.01, \omega^{2}=.09$. An analysis of simple main effects shows that, as 
predicted, when ability to process was high, argument quality exerted a stronger effect on the valenced message-related cognitive response index when the speaker used powerful language, $F(1,29)=37.75, p<.001, \omega^{2}=.60$; rather than powerless language, $F(1,27)=7.27, p<.05, \omega^{2}=.17$. Hence, Hypothesis $3 \mathrm{c}$ was supported.

However, the results of a three-way ANOVA with ability, argument quality, and language power as independent variables, and attitude as the dependent variable reveal that the Ability $\times$ Argument Quality $\times$ Language Power interaction was not significant $(F<1)$. In addition, in the high-ability condition, a two-way ANOVA yields a nonsignificant Argument Quality $\times$ Language Power interaction, $F(1,55)=1.02, p>.30$, offering no support for Hypothesis 3d.

\section{Discussion}

The results of the present study shed light on how style and substance work to affect persuasion. The data clearly support the multiple-roles perspective of language power's effects on persuasion, and they support ability to process as at least a partial determinant of the role that language power assumes in persuasion. The results indicate strongly that language power serves as a peripheral cue, and a distracting influence on persuasion. Less clear is the degree to which language power biases the processing of message arguments.

All four hypotheses postulating that language power acts as a peripheral cue found support in the present data. When message recipients were low in ability to cognitively respond to message arguments, powerless language negatively affected attitudes toward the advocated position. This result, consistent with some past research on language power (Sparks \& Areni, 2002; Sparks et al., 1998), suggests that if the ability to process message content is low, message recipients rely on cues such as powerless language markers to draw conclusions about the desirability of an advocated position.

Not surprisingly, powerless language exerted a negative effect on the valence of speaker-related thoughts. However, this result also supports language power's role as a peripheral cue. As a practical matter, when they are unable to process message content, message recipients may elaborate on speaker qualities. Those elaborations will be more negative in the presence of powerless language than in its absence. Additional support for this explanation comes from the lack of influence that language power exerted on the valence of message-related thoughts, the mediation of language power's effect on attitude by speaker-related thoughts, and the lack of similar mediation by message-related thoughts. 
The data also support language power as a distracting influence. When ability was high, powerless language directed participants' thoughts away from relevant arguments and toward the speaker, thus limiting the amount of elaboration available for the substance of the message. As such, when they were exposed to powerless language, participants generated more speakerrelated and fewer message-related thoughts than when they were exposed to powerful language. As a result, the effect of the argument-quality manipulation tended to abate in the powerless language condition, relative to the powerful language condition. However, the latter effect did not translate into actual attitudes toward the advocated position, perhaps because language power directly affected attitudes, which would tend to obscure the distraction effect.

Language power's role as a biasing influence received only equivocal support from the data. Attitudes were more negative in the presence of powerless speech markers than in their absence, lending support to the basic notion that biasing effects were present. However, the nature of the bias remains unclear. With high ability, we expected to find that the valenced message-related thought index, but not the speaker-related thought index, would mediate language power's effects on attitudes. Instead, the data largely contradicted our expectations. Message-related thoughts did not mediate language power's effect on attitude, but speaker-related thoughts did, even when ability was high.

This failure to observe a mediation of language power's effects on attitudes by message-related thinking may simply reflect the magnitude of the distraction effect under conditions of high ability to process. That is, if the distraction effect of powerless language is large enough, there will be little or no argument-related thinking. Hence, with respect to the valenced argument-related thought index, there is no point of comparison between the powerful and powerless language conditions (i.e., argument-related thinking cannot be more negative in the powerless language conditions because it simply does not occur). The substantial sizes of the effects of language power on the number of speaker-related thoughts $\left(\omega^{2}=.63\right)$ and argument-related thoughts $\left(\omega^{2}=.39\right)$ in the high-ability condition supports this interpretation.

Likewise, given Baron and Kenny's (1986) criteria for mediation, a very strong biasing effect would require substantial argument-related thinking in both the powerful and powerless language conditions. This, by definition, would preclude a strong distraction effect in the latter condition. Hence, given the measures used, combined with the limitations of ANCOVA for testing mediation effects, there is an inherent tradeoff between observing both the biasing effects and distraction effects of language power under conditions of high ability. Manipulations that create medium effect sizes for 
each influence would appear to be optimal for observing both effects in a single experiment.

However, given that previous studies have produced more convincing evidence for a biasing influence (e.g., Sparks \& Areni, 2002), language power might be considered a particularly formidable multiple-roles variable within the ELM. An audience that is either unwilling or unable to elaborate on the content of a message may permit language power to influence persuasion directly via the peripheral route using simple heuristics (i.e., "He sounds a little too uncertain, he can't be right.") to guide attitude formation or change. However, even for an audience both willing and able to consider the details of a message, language power still guides persuasion by distracting people from processing message content and then by biasing their assessment of relevant arguments and evidence to be consistent with their perception of the speaker's style.

An alternative interpretation of these results centers on Kruglanski's (Kruglanski \& Thompson, 1999; Thompson, Kruglanski, \& Speigel, 2000) unimodel of persuasion. The unimodel makes three basic assumptions pertinent to the reported effects of language power. First, there is no fundamental distinction between a peripheral cue and a central argument: Both influence persuasion via similar processes. Second, motivation and ability determine whether a given cue or argument will be processed at all, rather than whether it will influence persuasion. In other words, some cues are easily processed, while others require considerable effort; and the same is true for arguments. Motivation and ability influence overall effort, not underlying persuasion processes. Finally, the unimodel acknowledges that message recipients may have a variety of goals in processing a given communication, not simply the formation of accurate attitudes.

From a unimodel perspective, there is no inherent difference between using the speaker's expressed confidence (i.e., language power) or his or her specific attribute claims (i.e., argument quality) as a basis for determining attitude toward the advertised product. Either source of information is potentially useful for drawing inferences about the product. The languagepower manipulation may have had a greater influence under conditions of low and high ability simply because it was relatively easy to process. Participants only had to see a few nonverbal hesitations (e.g., "um," "ah") in the text to infer that the speaker lacked confidence in the low-power condition.

The result that the effects of language power on persuasion were mediated by speaker-related thoughts in both the low- and high-ability conditions is also consistent with the unimodel. For one thing, language power is embedded within the argument quality manipulation. The unimodel suggests that the Cue $\times$ Ability and Argument $\times$ Ability interactions typically observed in ELM research often depend on clear perceptual and temporal distinctions 
between cues and arguments in the message stimulus. When these distinctions are not obvious, as was the case with the language-power manipulation, the conditional relationships specified by the ELM do not necessarily hold (Kruglanski \& Thompson, 1999). In addition, participants were told that they would read a "verbatim transcript of a person giving a testimonial about a computer product" and that they would evaluate the testimonial in terms of its suitability for use in an actual advertisement. Given this set of instructions, it is hardly surprising that all persuasive effects of language power were mediated by thoughts about the speaker. This is essentially what participants were told to think about. Future research on language power and other persuasion variables would benefit from designs that distinguish between ELM and unimodel predictions (Kruglanski \& Thompson, 1999).

Whether one interprets these results in terms of the ELM or the unimodel, in the case of language power, style does appear to be more important than substance. As noted at the beginning of this discussion, the results of this and previous language-power research suggest numerous future research possibilities. A potentially fruitful research avenue concerns the degree to which salience or ability moderates language power's effects when stimuli are presented in audiovisual modes, rather than in writing. Sparks et al. (1998), for example, found differences in language-power effects across modes, but could not ascertain whether these differences arose because of differing processing abilities inherent in each mode or whether modes differed sufficiently in terms of salience to produce the effects they report.

Our use of written stimuli, apart from their benefits to internal validity, permitted us to focus on the specific issue of ability. Future research could isolate the effects of salience and ability across modes to determine which moderating effect is at work. Another area of future research relates to the effects of the embeddedness of communication variables. Our results imply that language power's embeddedness in the persuasive communication may have led message recipients to treat the language markers as if they were arguments. Future research could examine the degree to which embeddedness competes with or augments communication variables' relevance to message arguments.

\section{References}

Areni, C. S. (2002). The proposition-probability model of argument structure and message acceptance. Journal of Consumer Research, 29, 168-187. Areni, C. S. (2003). The effects of structural and grammatical variables on persuasion: An elaboration likelihood model perspective. Psychology and Marketing, 20, 349-375. 
Areni, C. S., \& Sparks, J. R. (2005). Language power and persuasion. Psychology and Marketing, 22, 507-525.

Baron, R. M., \& Kenny, D. A. (1986). The moderator-mediator variable distinction in social psychological research: Conceptual, strategic, and statistical considerations. Journal of Personality and Social Psychology, 51, 1173-1182.

Bradac, J. J., \& Mulac, A. (1984a). Attributional consequences of powerful and powerless speech styles in a crisis-intervention context. Journal of Language and Social Psychology, 3, 1-19.

Bradac, J. J., \& Mulac, A. (1984b). A molecular view of powerful and powerless speech styles: Attributional consequences of specific language features and communicator intentions. Communication Monographs, 51, 307-319.

Bradac, J. J., \& Street, R. L., Jr. (1990). Powerful and powerless styles of talk: A theoretical analysis of language and impression formation. Research in Language and Social Interaction, 23, 195-242.

Chaiken, S., \& Eagly, A. H. (1976). Communication modality as a determinant of message persuasiveness and message comprehensibility. Journal of Personality and Social Psychology, 34, 605-614.

Chaiken, S., \& Eagly, A. H. (1983). Communication modality as a determinant of persuasion: The role of communicator salience. Journal of Personality and Social Psychology, 45, 241-256.

Erickson, B., Lind, E. A., Johnson, B. C., \& O’Barr, W. M. (1978). Speech style and impression formation in a court setting: The effects of "powerful" and "powerless" speech. Journal of Experimental Social Psychology, 14, 266-279.

Gibbons, P., Busch, J., \& Bradac, J. J. (1991). Powerful versus powerless language: Consequences for persuasion impression formation, and cognitive response. Journal of Language and Social Psychology, 10, $115-133$.

Greenwald, A. G. (1968). Cognitive learning, cognitive response to persuasion, and attitude change. In A. G. Greenwald, T. C., Brock \& T. M. Ostrom (Eds.), Psychological foundations of attitudes (pp. 147-170). New York: Academic Press.

Heil, M., \& Rolke, B. (2004). Unattended distractor-induced priming in a visual selective attention task. Journal of Psychophysiology, 18, 164169.

Heller, E., \& Areni, C. S. (2004). The effects of conditional indicative language on the comprehension and acceptance of advertising claims. Journal of Marketing Communications, 10, 229-240.

Holtgraves, T., \& Lasky, B. (1999). Linguistic power and persuasion. Journal of Language and Social Psychology, 18, 196-205. 
Hosman, L. A., \& Wright, J. W., II (1987). The effects of hedges and hesitations on impression formation in a simulated courtroom context. Western Journal of Speech Communication, 51, 173-188.

Kruglanski, A. W., \& Thompson, E. P. (1999). Persuasion by a single route: A view from the unimodel. Psychological Inquiry, 10, 83-109.

MacInnis, D. J., Moorman, C., \& Jaworski, B. J. (1991). Enhancing and measuring consumers' motivation, opportunity, and ability to process brand information from ads. Journal of Marketing, 55, 32-53.

Maheswaran, D., \& Chaiken, S. (1991). Promoting systematic processing in low-motivation settings: Effect of incongruent information on processing and judgment. Journal of Personality and Social Psychology, 61, $13-25$.

Moore, D. L., Hausknecht, D., \& Thamodaran, K. (1986). Time compression, ability, and persuasion, Journal of Consumer Research, 13, 85-99.

Munch, J. M., Boller, G. W., \& Swasy, J. L. (1993). The effects of argument structure and affective tagging on product attitude formation. Journal of Consumer Research, 20, 294-302.

Munch, J. M., \& Swasy, J. L. (1988). Rhetorical questions, summarization frequency, and argument strength effects on recall. Journal of Consumer Research, 15, 69-76.

O'Barr, W. M. (1982). Linguistic evidence. New York: Academic Press.

Petty, R. E., \& Cacioppo, J. T. (1986). Communication and persuasion: Central and peripheral routes to attitude change. New York: SpringerVerlag.

Petty, R. E., Cacioppo, J. T., \& Goldman, R. (1981). Personal involvement as a determinant of argument-based persuasion. Journal of Personality and Social Psychology, 41, 847-855.

Petty, R. E., Cacioppo, J. T., \& Schumann, D. (1983). Central and peripheral routes to advertising effectiveness: The moderating role of involvement. Journal of Consumer Research, 10, 134-148.

Petty, R. E., Priester, J. R., \& Wegener, D. T. (1994). Cognitive processes in attitude change. In R. S. Wyter \& T. K. Srull (Eds.), Handbook of social cognition (2nd ed., pp. 69-142). Hillsdale, NJ: Lawrence Erlbaum.

Petty, R. E., Unnava, H. R., \& Strathman, A. J. (1991). Theories of attitude change. In T. S. Robertson \& H. H. Kassarjain (Eds.), Handbook of consumer behavior (pp. 241-280). Englewood Cliffs, NJ: Prentice Hall.

Petty, R. E., Wells, G. L., \& Brock, T. C. (1976). Distraction can enhance or reduce yielding to propaganda: Thought disruption versus effort justification. Journal of Personality and Social Psychology, 34, 874-884.

Sparks, J. R., \& Areni, C. S. (2002). The effects of sales presentation quality and initial perceptions on persuasion: A multiple-role perspective. Journal of Business Research, 55, 517-528. 
Sparks, J. R., Areni, C. S., \& Cox, K. C. (1998). An investigation of the effects of language power and communication modality on persuasion. Communication Monographs, 65, 108-125.

Thompson, E. P., Chaiken, S., \& Hazlewood, J. D. (1987). Need for cognition and desire for control as moderators of extrinsic reward effects: A person by situation approach to the study of intrinsic motivation. Journal of Personality and Social Psychology, 64, 987-999.

Thompson, E. P., Kruglanski, A. W., \& Speigel, S. (2000). Attitudes as knowledge structures and persuasion as a specific case of subjective knowledge acquisition. In G. R. Maio \& J. M. Olson (Eds.), Why we evaluate: Functions of attitudes (pp. 59-96). Mahwah, NJ: Lawrence Erlbaum.

Wheeler, S. C., Petty, R. E., \& Bizer, G. Y. (2005). Self-schema matching and attitude change: Situational and dispositional determinants of message elaboration. Journal of Consumer Research, 31, 787-797. 